\title{
On the complexity of routing permutations on trees by arc-disjoint paths (extended abstract)
}

\author{
D. Barth $^{\dagger}$, S. Corteel ${ }^{\ddagger}$, A. Denise ${ }^{\ddagger}$, D. Gardy ${ }^{\dagger}$, M. Valencia-Pabon ${ }^{\ddagger}$ \\ † PRiSM, Université de Versailles, 45 Av. des Etats Unis, 78035 VERSAILLES, FR. \\ $\ddagger$ L.R.I., Bât 490, Université Paris-Sud, 91405 ORSAY, FR.
}

\begin{abstract}
In this paper we show that the routing permutation problem is NP-hard even for binary trees. Moreover, we show that in the case of unbounded degree tree networks, the routing permutation problem is NP-hard even if the permutations to be routed are involutions. Finally, we show that the average-case complexity of the routing permutation problem on linear networks is $n / 4+o(n)$.
\end{abstract}

Keywords: Average-Case Complexity, Routing Permutations, Path Coloring, Tree Networks, NP-completeness.

\section{Introduction}

Efficient communication is a prerequisite to exploit the performance of large parallel systems. The routing problem on communication networks consists in the efficient allocation of resources to connection requests. In this network, establishing a connection between two nodes requires selecting a path connecting the two nodes and allocating sufficient resources on all links along the paths associated to the collection of requests. In the case of all-optical networks, data is transmitted on lightwaves through optical fiber, and several signals can be transmitted through a fiber link simultaneously provided that different wavelengths are used in order to prevent interference (wavelength-division multiplexing) [4]. As the number of wavelengths is a limited resource, then it is desirable to establish a given set of connection requests with a minimum number of wavelengths. In this context, it is natural to think in wavelengths as colors. Thus the routing problem for all-optical networks can be viewed as a path coloring problem: it consists in finding a desirable collection of paths on the network associated with the collection of connection requests in order to minimize the number of colors needed to color these paths in such a way that any two different paths sharing a same link of the network are assigned different colors. For simple networks, such as trees, the routing problem is simpler, as there is always a unique path for each communication request.

This paper is concerned with routing permutations on trees by arc-disjoint paths, that is, the path coloring problem on trees when the collection of connection requests represents a permutation of the nodes of the tree network.

Previous and related work. In [1], Aumann and Rabani have shown that $O\left(\frac{\log ^{2} n}{\beta^{2}}\right)$ colors suffice for routing any permutation on any bounded degree network on $n$ nodes, where $\beta$ is the arc expansion of the network. The result of 
Aumman and Rabani almost matches the existential lower bound of $\Omega\left(\frac{1}{\beta^{2}}\right)$ obtained by Raghavan and Upfal [18]. In the case of specific network topologies, Gu and Tamaki [13] proved that 2 colors are sufficient to route any permutation on any symmetric directed hypercube. Independently, Paterson et al. [17] and Wilfong and Winkler [22] have shown that the routing permutation problem on ring networks is NP-hard. Moreover, in [22] a 2-approximation algorithm is given for this problem on ring networks. To our knowledge, the routing permutation problem on tree networks by arc-disjoint paths has not been studied in the literature.

Our results. In Section 2 we first give some definitions and recall previous results. In Section 3 we show that for arbitrary permutations, the routing permutation problem is NP-hard even for binary trees. Moreover, we show that the routing permutations problem on unbounded degree trees is NP-hard even if the permutations to be routed are involutions, i.e. permutations with cycles of length at most two. In Section 4 we focus on linear networks. In this particular case, since the problem reduces to coloring an interval graph, the routing of any permutation is easily done in polynomial time [14]. We show that the average number of colors needed to color any permutation on a linear network on $n$ vertices is $n / 4+o(n)$. As far as we know, this is the first result on the average-case complexity for routing permutations on networks by arc-disjoint paths. Finally, in Section 5 we give some open problems and future work.

\section{Definitions and preliminary results}

We model the tree network as a rooted labeled symmetric directed tree $T=$ $(V, A)$, where processors and switches are vertices and links are modeled by two arcs in opposite directions. In the sequel, we assume that the labels of the vertices of a tree $T$ on $n$ vertices are $\{1,2, \ldots, n\}$ and are such that a postfix tree traversal would be exactly $1,2, \ldots, n$. This implies that for any internal vertex labeled by $i$ the labels of the vertices in his subtree are less than $i$. Given two vertices $i$ and $j$ of the tree $T$, we denote by $\langle i, j\rangle$ the unique path from vertex $i$ to vertex $j$. The arc from vertex $i$ to its father (resp. from the father of $i$ to $i$ ) $(1 \leq i \leq n-1)$ is labeled by $i^{+}$(resp. $i^{-}$). See Figure 1(a) for the linear network on $n=6$ vertices rooted at vertex $i=6$. We want to route permutations in $S_{n}$ on any tree $T$ on $n$ vertices. Given a tree $T$ and a vertex $i$ we call $T(i)$ the subtree of $T$ rooted at vertex $i$.

We associate with any permutation a graphical representation. To represent the permutation $\sigma$ we draw an arrow from $i$ to $\sigma(i)$, if $i \neq \sigma(i)$, that is, the path $<i, \sigma(i)>, 1 \leq i \leq n$. The arrow going from $i$ to $\sigma(i)$ crosses the arc $j^{+}$if and only if $i$ is in $T(j)$ and $\sigma(i)$ is not in $T(j)$ and it crosses the arc $j^{-}$if and only if $i$ is not in $T(j)$ and $\sigma(i)$ is in $T(j), 1 \leq j \leq n-1$.

Definition 1. Let $T$ be a tree on $n$ vertices and $\sigma$ be a permutation in $\mathcal{S}_{n}$. We define the height of the arc $i^{+}$(resp. height of the arc $i^{-}$), $1 \leq i \leq n-1$, denoted $h_{T}^{+}(\sigma, i)$ (resp. $h_{T}^{-}(\sigma, i)$ ), as the number of paths crossing the arc $i^{+}$ 


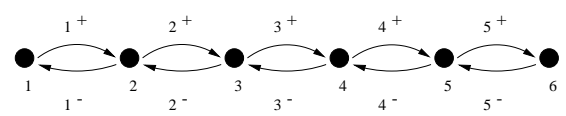

(a)

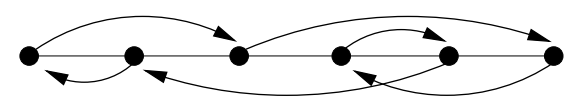

(b)

Fig. 1. (a) Labeling of the vertices and the arcs for the linear network on $n=6$ vertices rooted at vertex $i=6$. (b) representation of permutation $\sigma=(3,1,6,5,2,4)$ on the linear network given in (a).

(resp. $\left.i^{-}\right)$; that is, $h_{T}^{+}(\sigma, i)=|\{j \in T(i) \mid \sigma(j) \notin T(i)\}|\left(\right.$ resp. $h_{T}^{-}(\sigma, i)=\mid\{j \notin$ $T(i) \mid \sigma(j) \in T(i)\} \mid)$.

Lemma 1. Let $T$ be a tree with $n$ vertices. For all $\sigma$ in $\mathcal{S}_{n}$ and for all $i \in$ $\{1,2, \ldots, n-1\}, h_{T}^{+}(\sigma, i)=h_{T}^{-}(\sigma, i)$.

This lemma is straightforward to prove. It tells us that in order to study the height of a permutation on a tree on $n$ vertices, it suffices to consider only the height of the labeled $\operatorname{arcs} i^{+}$.

Definition 2. Given a tree $T$ and a permutation $\sigma$ to be routed on $T$, the height of $\sigma$, denoted $h_{T}(\sigma)$, is the maximum number of paths crossing any arc of $T$ : $h_{T}(\sigma)=\max _{i} h_{T}^{+}(\sigma, i)$.

For example the permutation $\sigma=(3,1,6,5,2,4)$ on the linear network in Figure 1(a) has height 2 (see Figure 1(b)). The maximum is reached in the $\operatorname{arcs} 4^{ \pm}$.

Definition 3. Given a tree $T$ and a permutation $\sigma$ to be routed on $T$, the coloration number of $\sigma$, denoted $R_{T}(\sigma)$, is the minimum number of colors assigned to the paths on $T$ associated with $\sigma$ such that no two paths sharing a same arc of $T$ are assigned the same color.

Clearly, for any permutation $\sigma$ of the vertex set of a tree $T$, we have $R_{T}(\sigma) \geq$ $h_{T}(\sigma)$. For linear networks the equality holds, because the conflict graph of the paths associated with $\sigma$ is an interval graph (see [12]). Moreover, optimal vertex coloring for interval graphs can be computed efficiently [14]. However, for arbitrary tree networks, equality does not hold as we will see in the Section 3.3.

\section{Complexity of computing the coloration number}

We begin this section by showing the NP-completeness of the routing permutations problem in binary trees, and then for the case of routing involutions on unbounded degree trees. Finally, we discuss some polynomial cases of this problem and we show, by an exemple, that in the case of binary trees having at most two vertices with degree equal to 3 , the equality between the height and the coloration number of permutations does not hold. 


\section{$3.1 \quad$ NP-completeness results}

Independently, Kumar et al. [15] and Erlebach and Jansen [6] have shown that computing a minimal coloring of any collection of paths on symmetric directed binary trees is NP-hard. However, the construction given in $[15,6]$ does not work when the collection of paths represents a permutation of the vertex set of a binary tree. Thus, by using a reduction similar to the one used in $[15,6]$ we obtain the following result.

Theorem 1. Let $\sigma \in S_{n}$ be any permutation to be routed on a symmetric directed binary tree $T$ on $n$ vertices, then computing $R_{T}(\sigma)$ is NP-hard.

Sketch of the proof. We use a reduction from the ARC-COLORING problem [19]. The ARC-COLORING problem can be defined as follows : given a positive integer $k$, an undirected cycle $C_{n}$ with vertex set numbered clockwise as $1,2, \ldots, n$, and any collection of paths $F$ on $C_{n}$, where each path $\langle v, w\rangle \in F$ is regarded as the path beginning at vertex $v$ and ending at vertex $w$ again in the clockwise direction, does $F$ can be colored with $k$ colors so that no two paths sharing an edge of $C_{n}$ are assigned the same color ? It is well known that the ARC-COLORING problem is NP-complete [10]. Let $I$ be an instance of the ARC-COLORING problem. We construct from $I$ an instance $I^{\prime}$ of the routing permutations problem on binary trees, consisting of a symmetric directed binary tree $T$ and a permutation-set of paths $F^{\prime}$ on $T$ such that $F$ can be $k$-colored if and only if $F^{\prime}$ can be $k$-colored. Without loss of generality, we may assume that each edge of $C_{n}$ is crossed by exactly $k$ paths in $F$. If some edge of $C_{n}$ is crossed by more than $k$ paths, then this can be discovered in polynomial time, and it implies that the answer in this instance $I$ must be "no". If some edge $[i, i+1]$ of $C_{n}$ is crossed by $r<k$ paths, then we can add $k-r$ paths of the form $\langle i, i+1\rangle$ (or $\langle i, 1\rangle$ if $i=n$ ) to $F$ without changing its $k$-colorability.

Let $B(i) \subset F$ (resp. $E(i) \subset F$ ) be the subcollection of paths of $F$ beginning (resp. ending) at vertex $i$ of $C_{n}, 1 \leq i \leq n$. Thus, by the previous hypothesis, it is easy to verify that the following property holds for instance $I$.

Claim. For all vertices $i$ of $C_{n},|B(i)|=|E(i)|$.

Construction of the binary tree $T$ of $I^{\prime}$ : first, construct a line on $2 k+n$ vertices denoted from left to right by $l_{k}, l_{k-1}, \ldots, l_{2}, l_{1}, v_{1}, v_{2}, \ldots, v_{n}, r_{1}, r_{2}, \ldots, r_{k}$. Next, for each vertex $l_{i}$ (resp. $\left.r_{i}\right), 1 \leq i \leq k$, construct a new different line on $2 k+1$ vertices denoted from left to right by $l l_{i}^{1}, l l_{i}^{2}, \ldots, l l_{i}^{k}, w l_{i}, r l_{i}^{k}, r l_{i}^{k-1}, \ldots, r l_{i}^{1}$ (resp. $\left.l r_{i}^{1}, l r_{i}^{2}, \ldots, l r_{i}^{k}, w r_{i}, r r_{i}^{k}, r r_{i}^{k-1}, \ldots, r r_{i}^{1}\right)$ and add to $T$ the arc set $\left\{\left(w l_{i}, l_{i}\right)\right.$, $\left.\left(l_{i}, w l_{i}\right)\right\}$ (resp. $\left.\left\{\left(w r_{i}, r_{i}\right),\left(r_{i}, w r_{i}\right)\right\}\right)$. Finally, for each vertex $v_{i}, 1 \leq i \leq n$, if $|B(i)|>1$, then construct a new different line on $\alpha_{i}=|B(i)|-1$ vertices denoted by $v_{i}^{1}, v_{i}^{2}, \ldots, v_{i}^{\alpha_{i}}$ and add to $T$ the arc set $\left\{\left(v_{i}^{1}, v_{i}\right),\left(v_{i}, v_{i}^{1}\right)\right\}$.

Construction of the permutation-set of paths $F^{\prime}$ of $I^{\prime}$ : for each path $\langle i, j\rangle \in F$, let $b_{i}\left(\right.$ resp. $\left.e_{j}\right)$ be the first vertex of $T$ in $\left\{v_{i}, v_{i}^{1}, \ldots, v_{i}^{\alpha_{i}}\right\}$ (resp. $\left\{v_{j}, v_{j}^{1}, \ldots, v_{j}^{\alpha_{j}}\right\}$ ) not already used by any path in $F^{\prime}$ as beginning-vertex (resp. ending-vertex), then we consider the following two types of paths in $F$ :

- Type $1: i<j$. Then add to $F^{\prime}$ the path set $\left\{\left\langle b_{i}, e_{j}\right\rangle\right\}$. 
- Type $2: i>j$. Let $r_{p}$ (resp. $l_{q}$ ) be the first vertex of $T$ in $\left\{r_{1}, r_{2}, \ldots, r_{k}\right\}$ (resp. $\left.\left\{l_{1}, l_{2}, \ldots, l_{k}\right\}\right)$ such that the arc $\left(r_{p}, w r_{p}\right)$ (resp. $\left.\left(l_{q}, w l_{q}\right)\right)$ of $T$ has not be already used by any path in $F^{\prime}$, then add to $F^{\prime}$ the path set $\left\{\left\langle b_{i}, r r_{p}^{1}\right\rangle,\left\langle l r_{p}^{1}, r l_{q}^{1}\right\rangle\right.$, $\left.<l l_{q}^{1}, e_{j}>\right\}$. In addition, for each $i, 1 \leq i \leq k$, add to $F^{\prime}$ the following path sets : $\left.\left\{<l l_{i}^{j}, r l_{i}^{j}\right\rangle: 2 \leq j \leq k\right\} \cup\left\{\left\langle r l_{i}^{s}, l l_{i}^{s}\right\rangle: 1 \leq s \leq k\right\}$ and $\left\{\left\langle l r_{i}^{j}, r r_{i}^{j}\right\rangle: 2 \leq j \leq\right.$ $k\} \cup\left\{\left\langle r r_{i}^{s}, l r_{i}^{s}\right\rangle: 1 \leq s \leq k\right\}$. The paths $\left\langle l l_{i}^{j}, r l_{i}^{j}\right\rangle$ and $\left\langle l r_{i}^{j}, r r_{i}^{j}\right\rangle, 2 \leq j \leq k$, $1 \leq i \leq k$, act as blockers. They make sure that all the three paths in $F^{\prime}$ corresponding to one path in $F$ of type 2 are colored with the same color in any $k$-coloration of $F^{\prime}$. The other paths that we call permutation paths, are used to ensure that the path collection $F^{\prime}$ represents a permutation of the vertex set of $T$. In Figure 2 we present an example of this polynomial construction. By

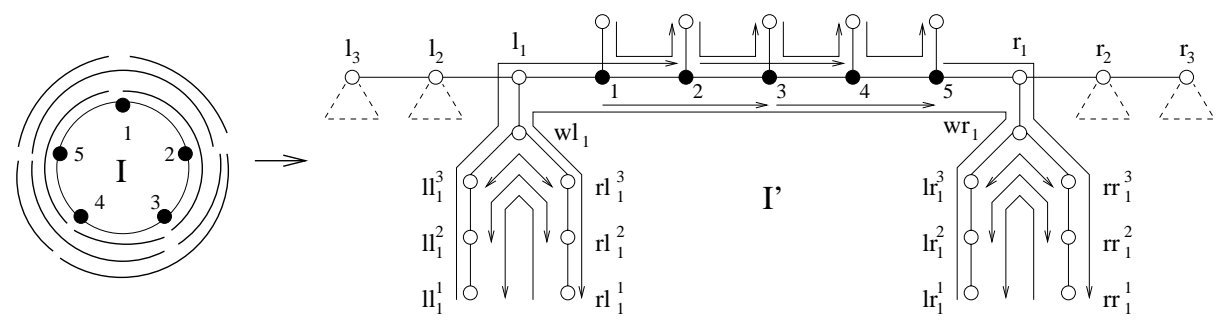

Fig. 2. Partial construction of $I^{\prime}$ from $I$, where $k=3$.

our construction, it is easy to check that the set of paths $F^{\prime}$ on $T$ represents a permutation of the vertex set of $T$, and that there is a $k$-coloring of $F$ if and only if there is a $k$-coloring of $F^{\prime}$.

In the case of unbounded degree symmetric directed trees, Caragiannis et al. [3] have shown that the path coloring problem remains NP-hard even if the collection of paths is symmetric (we call this problem the symmetric path coloring problem), i.e., for each path beginning at vertex $v_{1}$ and ending at vertex $v_{2}$, there also exists its symmetric, a path beginning at $v_{2}$ and ending at $v_{1}$. Thus, using a polynomial reduction from the symmetric path coloring problem on trees [3] we have the following result which proof is omitted for lack of space.

Theorem 2. Let $\sigma \in I_{n}$ be any involution to be routed on an unbounded degree tree $T$ on $n$ vertices. Then computing $R_{T}(\sigma)$ is NP-hard.

\subsection{Polynomial cases}

As noticed in Section 2, the coloration number associated to any permutation to be routed on a linear network can be computed efficiently in polynomial time [14]. In the case of generalized star networks, i.e., a tree network having only one vertex with degree greater to 2 and the other vertices with degree at most equal to 2, Gargano et al. [11] show that an optimal coloring of any collection of paths on these networks can be computed efficiently in polynomial time. Moreover, in [11] 
is also showed that the number of colors needed to color any collection of paths on a generalized star network is equal to the height of such a collection of paths. Thus, based on the results given in [11] we obtain the following proposition.

Proposition 1. Given a generalized star network $G$ on $n$ vertices and a permutation $\sigma \in S_{n}$ to be routed on $G$, the coloration number $R_{G}(\sigma)$ can be computed efficiently in polynomial time. Moreover, $R_{G}(\sigma)=h_{G}(\sigma)$ always holds.

\subsection{General trees}

Given any permutation $\sigma \in S_{n}$ to be routed on a tree $T$ on $n$ vertices, the equality between the heigth $h_{T}(\sigma)$ and the coloration number $R_{T}(\sigma)$ does not always hold. In Figure 3(a) we give an exemple of a permutation $\sigma \in S_{10}$ to be routed on a tree $T$ on 10 vertices, which height $h_{T}(\sigma)$ is equal to 2. Moreover, in Figure 3(b) we present the conflict graph $G$ associated with $\sigma$, that is an undirected graph whose vertices are the paths on $T$ associated with $\sigma$, and in which two vertices are adjacent if and only if their associated paths share a same arc in $T$. Thus, clearly the coloration number $R_{T}(\sigma)$ is equal to the chromatic number of $G$. Therefore, as the conflict graph $G$ has the cycle $C_{5}$ as induced subgraph, then the chromatic number of $G$ is equal to 3 , and thus $R_{T}(\sigma)=3$.

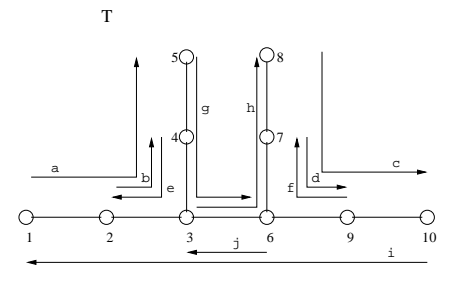

(a)

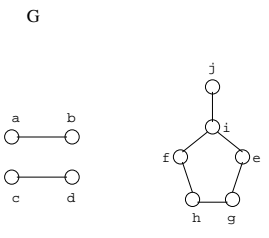

(b)

Fig. 3. (a) A tree $T$ on 10 vertices and a permutation $\sigma=(5,4,8,2,6,3,9,10,7,1)$ to be routed on $T$. (b) The conflict graph $G$ associated with permutation $\sigma$ in (a).

The best known approximation algorithm for coloring any collection of paths with height $h$ on any tree network is given in [7], which uses at most $\left\lceil\frac{5}{3} h\right\rceil$ colors. Therefore it trivially also holds for any permutation-set of paths with height $h$ on any tree.

Proposition 2. Given a tree $T$ on $n$ vertices and a permutation $\sigma \in S_{n}$ to be routed on $T$ with heigth $h_{T}(\sigma)$, there exists a polynomial algorithm for coloring the paths on $T$ associated with $\sigma$ which uses at most $\left\lceil\frac{5}{3} h_{T}(\sigma)\right\rceil$ colors.

\section{Average coloration number on linear networks}

The main result of this section is the following: 
Theorem 3. The average coloration number of the permutations in $S_{n}$ to be routed on a linear network on $n$ vertices is

$$
\frac{n}{4}+\frac{\lambda}{2} n^{1 / 3}+O\left(n^{1 / 6}\right)
$$

where $\lambda=0.99615 \ldots$

To prove this result, we use the equality between the height and the coloration number (see Section 2). Then our approach, developed in Subsections 4.1 and 4.2 , is as follows: at first we recall a bijection between permutations in $S_{n}$ and special walks in $\mathbb{N} \times \mathbb{N}$, called "Motzkin walks", which are labeled in a certain way. The bijection is such that the height parameter is "preserved". Then we prove Theorem 3 by studying the asymptotic behaviour of the height of these walks. On the other hand, we get in Subsection 4.3 the generating function of permutations with coloration number $k$, for any given $k$. This gives rise to an algorithm to compute exactly the average coloration number of the permutations for any fixed $n$.

\subsection{A bijection between permutations and Motzkin walks}

A Motzkin walk of length $n$ is a $(\mathrm{n}+1)$-uple $\left(s_{0}, s_{1}, \ldots, s_{n}\right)$ of points in $\mathbb{N} \times \mathbb{N}$ satisfying the following conditions:

- For all $0 \leq i \leq n, s_{i}=\left(i, y_{i}\right)$ with $y_{i} \geq 0$;

$-y_{0}=y_{n}=0$

- For all $0 \leq i<n, y_{i+1}-y_{i}$ equals either 1 (North-East step), or 0 (East step), or -1 (South-East step);

The height of a Motzkin walk $\omega$ is $H(\omega)=\max _{i \in\{0,1, \ldots, n\}}\left\{y_{i}\right\}$.

Labeled Motzkin walks are Motzkin walks in which steps can be labeled by integers. These structures are in relation with several well-studied combinatorial objects $[8,20,21]$ and in particular with permutations. The walks we will deal with are labeled as follows:

- each South-East step $\left(i, y_{i}\right) \rightarrow\left(i+1, y_{i}-1\right)$ is labeled by an integer between 1 and $y_{i}{ }^{2}$ (or, equivalently, by a pair of integers, each one between 1 and $y_{i}$ );

- each East step $\left(i, y_{i}\right) \rightarrow\left(i+1, y_{i}\right)$ is labeled by an integer between 1 and $2 y_{i}+1$.

Let $P_{n}$ be the set of such labeled Motzkin walks of length $n$. We recall that $\mathcal{S}_{n}$ is the set of permutations on $[n]$. The following result was first established by Françon and Viennot [9]:

Theorem (Françon-Viennot) There is a one-to-one correspondence between the elements of $P_{n}$ and the elements of $\mathcal{S}_{n}$.

Several bijective proofs of this theorem are known. Biane's bijection [2] is particular, in the sense that it preserves the height: to any labeled Motzkin walk of 
length $n$ and height $k$ corresponds a permutation in $S_{n}$ with height $k$ (and so with coloration number $k$ ). We do not present here the whole Biane's bijection; we just focus on the construction of the (unlabelled) Motzkin walk associated to a permutation, in order to show that the height is preserved. This property, which is not explicitely noticed in Biane's paper, is essential for our purpose.

Biane's correspondence between a permutation $\sigma=(\sigma(1), \sigma(2), \ldots, \sigma(n))$ and a labeled Motzkin walk $\omega=\left(s_{0}, s_{1}, \ldots, s_{n}\right)$ is such that, for $\left.1 \leq i \leq n\right)$ :

- step $\left(s_{i-1}, s_{i}\right)$ is a North-East step if and only if $\sigma(i)>i$ and $\sigma^{-1}(i)>i$;

$-\operatorname{step}\left(s_{i-1}, s_{i}\right)$ is a South-East step if and only if $\sigma(i)<i$ and $\sigma^{-1}(i)<i$;

- otherwise, step $\left(s_{i-1}, s_{i}\right)$ is an East step.

Now, for any $1 \leq i \leq n$, the height of point $s_{i}$ in $\omega$ is obviously equal to the number of North-East steps minus the number of South-East steps in the shrinked walk $\left(s_{0}, s_{1}, \ldots, s_{i}\right)$. On the other hand, we can prove easily that the height of arc $i^{+}$in $\sigma$ is equal to the number of integers $j \leq i$ such that $\sigma(j)>j$ and $\sigma^{-1}(j)>j$, minus the number of integers $j \leq i$ such that $\sigma(j)<j$ and $\sigma^{-1}(j)<j$. This proves the property. We present in Figure 4 an exemple of correspondence. The above description permits to construct the "skeleton" of the permutation, in the center of the figure, given the Motzkin walk on the top. Then the labeling of the path allows to complete the permutation. This is described in detail in [2] and in the full version of this paper, in preparation.

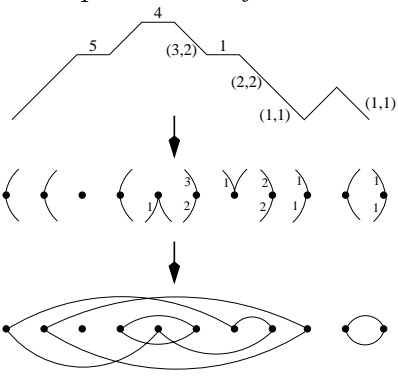

Fig. 4: From a walk to a permutation

\subsection{Proof of Theorem 3}

In [16], Louchard analyzes some list structures; in particular his "dictionary structure" corresponds to our labeled Motzkin walks. We will use his notation in order to refer directly to his article. From Louchard's theorem 6.2, we deduce the following lemma:

Lemma 2. The height $Y^{*}([n v])$ of a random labeled Motzkin walk of length $n$ after the step $[n v](v \in[0,1]))$ has the following behavior

$$
\frac{Y^{*}([n v])-n v(1-v)}{\sqrt{n}} \Rightarrow X(v)
$$

where " $\Rightarrow$ " denotes the weak convergence and $X$ is a Markovian process with mean 0 and covariance $C(s, t)=2 s^{2}(1-t)^{2}, s \leq t$.

Then the work of Daniels and Skyrme [5] gives us a way to compute the maximum of $Y^{*}([n v])$, that is the height of a random labeled Motzkin walk.

Proposition 3. The height of a random labeled Motzkin walk $Y^{*}$ is

$$
\max _{v} Y^{*}([n v])=\frac{n}{4}+m \sqrt{n / 2}+O\left(n^{1 / 6}\right),
$$


where $m$ is asymptotically Gaussian with mean $E(m) \sim \lambda n^{-1 / 6}(1 / 2)^{1 / 2}$ and variance $\sigma^{2}(m) \sim 1 / 8$ and $\lambda=0.99615 \ldots$

In the formula (1) of the above Proposition 2, the only non-deterministic part is $m$ which is Gaussian. So we just have to replace $m$ by $E(m)$ to prove Theorem 3.

\subsection{An algorithm to compute exactly the average coloration number}

We just have to look at known results in enumerative combinatorics [8,21] to get the generating function of the permutations of coloration number exactly $k$, that is

$$
\frac{(k !)^{2} z^{2 k}}{P_{k+1}^{*}(z) P_{k}^{*}(z)}
$$

with $P_{0}(z)=1, P_{1}(z)=z-b_{0}$ and $P_{n+1}(z)=\left(t-b_{n}\right) P_{n}(z)-\lambda_{n} P_{n-1}(z)$ for $n \geq 1$, where $P^{*}$ is the reprocical polynomial of $P$, that is $P_{n}^{*}(z)=z^{n} P_{n}(1 / z)$ for $n \geq 0$.

This generating function leads to a recursive algorithm to compute the number of permutations with coloration number $k$, denoted by $h_{n, k}$.

Proposition 4. The number of permutations in $\mathcal{S}_{n, k}$ follows the following recurrence

$$
h_{n, k}=\left\{\begin{array}{lr}
0 & \text { if } n<2 k \\
(k !)^{2} & \text { if } n=2 k \\
-\sum_{i=1}^{2 h+1} p(i) h_{n-i, k} \text { otherwise }
\end{array}\right.
$$

where $p(i)$ is the coefficient of $z^{i}$ in $P_{k+1}^{*}(z) P_{k}^{*}(z)$.

From this result we are able to compute the average height of a permutation as it is $\bar{h}(n)=\sum_{k \geq 0} k h_{n, k} / n$ !.

\section{Open problems and future work}

It remains open the complexity of routing involutions on binary trees by arcdisjoint paths. The average coloration number of permutations to be routed on general trees is also an interesting open problem. Computing the average coloration number of permutations to be routed on arbitrary topology networks seems a very difficult problem.

Acknowledgements. We are very grateful to Philippe Flajolet, Dominique Gouyou-Beauchamps and Guy Louchard for their help. 


\section{References}

1. Y. Aumann, Y. Rabani. Improved bounds for all optical routing. In Proc. of the 6th ACM-SIAM SODA, pp 567-576, 1995.

2. Ph. Biane. Permutations suivant le type d'excédance et le nombre d'inversions et interprétation combinatoire d'une fraction continue de Heine. Eur. J. Comb., 14(4):277-284, 1993.

3. I. Caragiannis, Ch. Kaklamanis, P. Persiano. Wavelength Routing of Symmetric Communication Requests in Directed Fiber Trees. In Proc. of SIROCCO, 1998.

4. N. K. Cheung, K. Nosu, and G. Winzer, editors. Special Issue on Dense Wavelength Division Multiplexing Techniques for High Capacity and Multiple Access Communications Systems. IEEE J. on Selected Areas in Comm., 8(6), 1990.

5. H. E. Daniels, T. H. R. Skyrme. The maximum of a random walk whose mean path has a maximum. Adv. Appl. Probab., 17:85-99, 1985.

6. T. Erlebach and K. Jansen. Call scheduling in trees, rings and meshes. In Proc. of HICSS-30, vol. 1, pp 221-222. IEEE CS Press, 1997.

7. T. Erlebach, K. Jansen, C. Kaklamanis, M. Mihail, P. Persiano. Optimal wavelength routing on directed fiber trees. Theoret. Comput. Sci. 221(1-2):119-137, 1999.

8. Ph. Flajolet. Combinatorial aspects of continued fractions. Discrete Math., 32:125$161,1980$.

9. J. Françon, X. Viennot. Permutations selon leurs pics, creux, doubles montées et doubles descentes, nombres d'Euler et nombres de Genocchi. Discrete Math., 28:2135, 1979.

10. M.R. Garey, D.S. Johnson, G.L. Miller, C.H. Papadimitriou. The complexity of colouring circular arcs and chords. SIAM J. Alg. Disc. Meth., 1(2):216-227,1980.

11. L. Gargano, P. Hell, S. Perennes. Coloring all directed paths in a symmetric tree with applications to WDM routing. In Proc. of ICALP, LNCS 1256, pp 505-515, 1997.

12. M. C. Golumbic. Algorithmic graph theory and perfect graphs. Academic Press, New York, 1980.

13. Q.-P. Gu, H. Tamaki. Routing a permutation in the Hypercube by two sets of edgedisjoint paths. In Proc. of 10th IPPS. IEEE CS Press, 1996.

14. U. I. Gupta, D. T. Lee, J. Y.-T. Leung. Efficient algorithms for interval graphs and circular-arc graphs. Networks, 12:459-467, 1982.

15. S. R. Kumar, R. Panigrahy, A. Russel, R. Sundaram. A note on optical routing on trees. Inf. Process. Lett., 62:295-300, 1997.

16. G. Louchard. Random walks, Gaussian processes and list structures. Theoret. Comput. Sci., 53:99-124, 1987.

17. M. Paterson, H. Schröder, O. Sýkora, I. Vrto. On Permutation Communications in All-Optical Rings. In Proc. of SIROCCO, 1998.

18. P. Raghavan, U. Upfal. Efficient routing in all optical networks. In Proc. of the 26th ACM STOC, pp 133-143, 1994.

19. A. Tucker. Coloring a family of circular arcs. SIAM J. Appl. Maths., 29(3):493-502, 1975.

20. X. Viennot. A combinatorial theory for general orthogonal polynomials with extensions and applications. Lect. Notes Math., 1171:139-157, 1985. Polynômes orthogonaux et applications, Proc. Laguerre Symp., Bar-le-Duc/France 1984.

21. X. Viennot. Une théorie combinatoire des polynômes orthogonaux généraux. Notes de conférences, Univ. Quebec, Montréal, 1985.

22. G. Wilfong, P. Winkler. Ring routing and wavelength translation. In Proc. of the 9th Annual ACM-SIAM SODA, pp 333-341, 1998. 